\title{
Electromagnetic Signals at RHIC
}

\author{
Simon Turbide and Charles Gale
}

Department of Physics, McGill University, 3600 University Street, Montreal, Canada H3A 2T8

\begin{abstract}
We calculate the direct photon yield in central and mid-peripheral $\mathrm{Au}+\mathrm{Au}$ collisions at the Relativistic Heavy-Ion Collider (RHIC) $\left(\sqrt{S_{N N}}=200 \mathrm{GeV}\right)$. The processes involving the propagation of jets have been convolved with a leading order treatment of jet energy loss in the medium and a one dimensional hydrodynamic expansion. The quark-gluon plasma (QGP) contribution turns out to be important, especially the in-medium conversion of a jet into a photon, for successfully describing recent photon measurements.
\end{abstract}

The search for the creation of a quark-gluon plasma (QGP) in relativistic heavy ion collisions has driven many experimental and theoretical investigations. Electromagnetic radiation (real and virtual photons), as it does not suffer final state interactions, constitutes a class of very interesting probes of the hot and dense medium created during those collisions. Indeed, they carry to the detectors information about the state of the system at the time they have been produced. If a hot thermalized phase is created in nucleusnucleus collisions, we thus expect to see in the photon spectrum, after subtracting the background coming from the decay of neutral mesons, some trace of these thermal photons.

The first direct photon measurements for $\mathrm{Pb}(158 \mathrm{~A} \mathrm{GeV})+\mathrm{Pb}$ at the Super Proton synchrotron (SPS) have been done by the WA98 collaboration [1]. Our previous calculations [2] assert that with a moderate value of the initial temperature $\left(T_{i}=205 \mathrm{MeV}\right)$, there was no strong evidence of the formation of the QGP. In other words, a contribution from the QGP was not crucial to a successful interpretation of the data. At RHIC energies, however, the discovery of high $p_{T}$ suppression in hadron spectra [3] is highly suggestive of the formation of a hot and dense phase. This represents new physics, over that of the SPS. The most currently plausible explanation for this observation is the energy loss of jets by induced gluon bremsstrahlung, as they go through a hot and dense partonic phase. In analogy with the jets interacting strongly with the partonic phase, it has been suggested in [4] that jets could interact electromagnetically with the medium as well. It was found that the direct annihilation and Compton scattering of quark jets, with incoming thermal partons, was an important source of photons at intermediate $p_{T}$. However, jet energy loss still needed to be included consistently.

Before a comparison with experimental data, we briefly present the different contributions to the direct photon spectrum. Firstly, direct photons from primary hard Compton and annihilation processes, at the partonic level, $a+b \rightarrow c+\gamma$, are produced during the overlap of the nuclei, with no final state effect. Secondly, jets also produced during the initial early stages $(a+b \rightarrow c+d)$, can thereafter go through the medium, lose some energy by gluon bremsstrahlung $\left(c \rightarrow c^{*}+g\right)$, and fragment into photons outside the 
medium $\left(c^{*} \rightarrow c^{*}+\gamma\right)$. This jet-fragmentation scenario can be expressed by

$$
\frac{d N^{j e t-f r a g}}{d^{2} p_{T} d y}=\sum_{f} \frac{d N^{f}}{d^{2} p_{T} d y} \otimes D_{f / \gamma}\left(z, p_{T}\right)
$$

where $D_{f / \gamma}$ is the photon fragmentation function, and $d N^{f} / d^{2} p_{T} d y$ is the momentum distribution of the parton of flavour $f$, after it has crossed the hot medium. The path length dependence of this distribution is obtained with the complete leading order description of Arnold, Moore and Yaffe (AMY) [5]. The primary hard direct photons added to the photons from fragmentation, are defined to be the prompt photons.

As previously mentioned, the interaction of jets with the medium can also produce photons by direct scattering (jet $+i \rightarrow j+\gamma$ ), where $(i, j)$ stand for thermal partons, but also through medium-induced photon bremsstrahlung. The photon yield generated by those jet-induced mechanisms can be written by

$$
\frac{d N^{\text {jet-induced }}}{d^{2} p_{T} d y}=\int d t \int d^{3} x \int d^{3} q \frac{d N^{q}}{d^{2} q_{T} d y^{\prime}}(t) \otimes \sigma_{j e t(q) \rightarrow \gamma(p)}^{\text {jet-induced }}
$$

The cross-section for the direct process is dominated by transfer of the entire jet momentum to the photon, such that $\sigma_{\text {jet }(q) \rightarrow \gamma(p)}^{\text {jet-th }} \propto \delta^{3}(q-p)$ : this process is labeled the jet-photon conversion. Details about these mechanisms are given in Ref. [6]. Finally, the medium can also simply radiate photons. Calculations for the QGP and hadronic gas induced radiations can be found in Refs. [7] and [2] respectively.

The work here differs from that of Ref. [6], as we have extended our direct photon calculations to non-central collisions. This is achieved by the following three steps. (1) The initial jet spectrum is scaled with the overlap factor of the nuclei: $d N^{f}(b)=$ $d N^{f}(b=0) T_{A B}(b) / T_{A B}(b=0)$. (2) The thermalized medium evolves according to a 1D Bjorken [8] expansion with the initial conditions given by $\tau_{i} T_{i}^{3} \propto d N(b) / d y / A_{\perp}(b)$, where $d N / d y$ is the particle rapidity density, and $A_{\perp}$ is the overlap area of the nuclei in the transverse plane. (3) Finally, the overlap zone of the nuclei has an "almond" shape, such that the total energy lose by a jet will depend on its direction.

Our photon yield calculations for $\mathrm{Au}+\mathrm{Au}$ collisions at RHIC are shown in Fig. 1 For a fixed initial time $\tau_{i}=0.26 \mathrm{fm} / \mathrm{c}$, we use the initial temperatures $T_{i}=370$ [2] and $340 \mathrm{MeV}$ for central (0-20\%) and mid-peripheral (30-40\%) collisions respectively. We define by QGP all photons which have been produced during the QGP phase. For central collisions, we reproduce the preliminary data from PHENIX [9] only when the QGP contributions are included, with our initial temperature, while we are below the data by a factor $\sim 2$ around $p_{T}=3 \mathrm{GeV}$, when all QGP contributions, except the jet-photon conversion, are included. For the $30-40 \%$ centrality class (right panel), the PHENIX data [10] show good precision only at high $p_{T}$, such that we have not included the hadronic contribution, which is not expected to contribute much in this range. While the high $p_{T}$ spectrum seems to be fully dominated by prompt photons, our calculations show again an important contribution from the QGP in the intermediate $p_{T}$ window. In proportion, this contribution is however $25 \%$ less important than for the central class, as we might have expected, since the initial temperature and the size of the interacting zone both decrease with increasing impact parameter. 


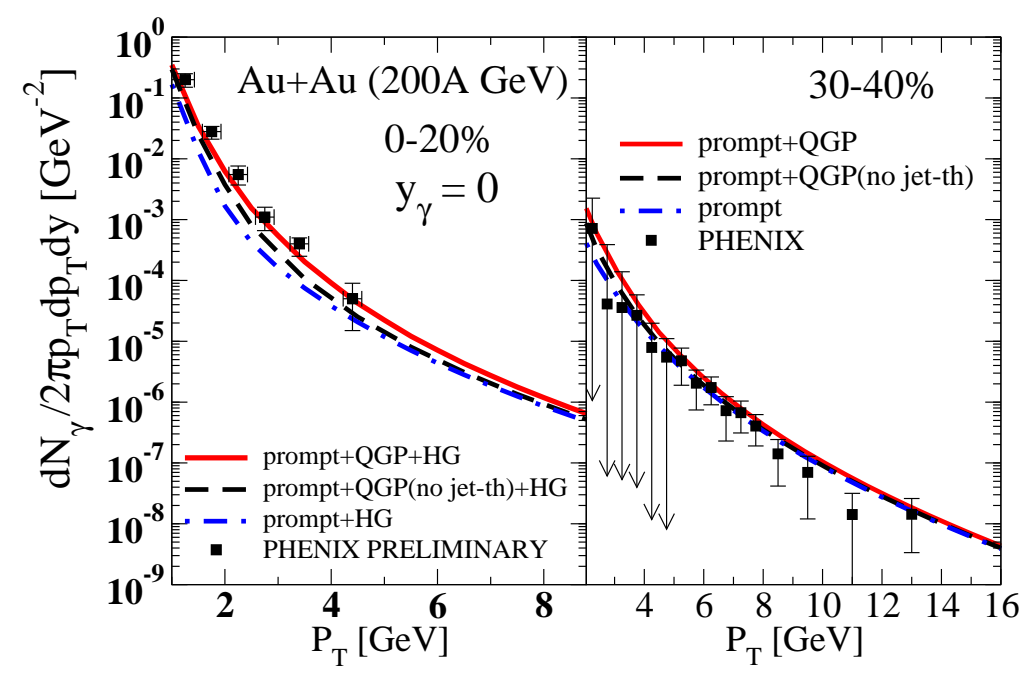

FIGURE 1. Direct photon spectrum at midrapidity, in Au+Au collision at RHIC. Left hand side: solid line, all direct photons; dashed line, all direct photon without the jet-photon conversion; and dot-dashed line, all direct photons without a QGP contribution; compared to PHENIX data [9] for a 0-20\% centrality class. Right hand side: solid line, prompt and QGP photons; dashed line, prompt and QGP without the jet-photon conversion; and dot-dashed line, prompt photons only; compared to PHENIX data [10] for a $30-40 \%$ centrality class.

In conclusion, we argue that the QGP contributions are important, particularly the jetphoton conversion, to the interpretation of the experimental data on direct photons. We expect virtual photons to exhibit a similar feature: this topic is presently under study.

\section{ACKNOWLEDGMENTS}

We thank S. Jeon and G.D. Moore for helpful discussions. This work was supported in part by the Natural Sciences and Engineering Research Council of Canada, and in part by le Fonds Nature et Technologies du Québec.

\section{REFERENCES}

1. M. M. Aggarwal et al., WA98 Collaboration, Phys. Rev. Lett. 85, 3595 (2000).

2. S. Turbide, R. Rapp, and C. Gale, Phys. Rev. C 69, 014903 (2004).

3. S.S. Adler et al., Phys. Rev. Lett. 91, 072301 (2003).

4. R.J. Fries, B. Müller and D.K. Srivastava, Phys. Rev. Lett 90, 132301 (2003).

5. P. Arnold, G. D. Moore and L. Yaffe, JHEP 0111, 057 (2001); JHEP 0206, 030 (2002).

6. S. Turbide, C. Gale, S. Jeon and G. D. Moore, Phys. Rev. C 72, 014906 (2005).

7. P. Arnold, G.D. Moore and L.G. Yaffe, J. High-Energy Phys. 0012, 009 (2001).

8. J.D. Bjorken, Phys. Rev. D 27, 140 (1983).

9. S. Bathe, talk given at Quark Matter 2005, Budapest, Hungary (2005).

10. S.S. Adler et al., Phys. Rev. Lett. 94, 232301 (2005). 